\title{
Physician engagement: the Vancouver Medical Staff Association engagement charter
}

\author{
Authors: Simon W Rabkin, ${ }^{\mathrm{A}}$ Marshall Dahl, ${ }^{\mathrm{B}}$ Ryan Patterson, ${ }^{\mathrm{C}}$ Noa Mallek, ${ }^{\mathrm{D}}$ Lynn Straatman, ${ }^{\mathrm{E}}$ Andrew Pinfold, ${ }^{\mathrm{F}}$ \\ Marthe Kenny Charles, ${ }^{\mathrm{G}}$ Stephen van Gaal, ${ }^{\mathrm{H}}$ Sophia Wong ${ }^{\mathrm{I}}$ and Himat Vaghadia ${ }^{\mathrm{J}}$
}

Engagement of physicians with their healthcare community or institution should be a central issue in healthcare because it can be translated into improved patient care, enhanced well-being for physicians as well as safer, more effective and less costly healthcare. To accomplish the mission/goal of meaningful physician engagement, we set about to establish a 'charter' for physician engagement. We defined our concept of meaningful physician engagement and customised the engagement spectrum construct for physician relationship with their healthcare community or institution. While recognising the importance of physician leaders within the hierarchical system for efficacy of organisational management, relying only on physicians in formal executive positions is insufficient for developing physician engagement. There is a need for widespread physician engagement across the organisation. The objective is both an improvement in patient care and in physician well-being.

Authors: ${ }^{\text {A }}$ rofessor of medicine, Vancouver General Hospital, Vancouver, Canada and president of medical staff, Vancouver Medical, Dental and Allied Staff Association, Vancouver, Canada; ${ }^{B}$ Clinical professor of medicine, University of British Columbia and Vancouver Hospital, Vancouver, Canada and former president of medical staff, Vancouver Medical, Dental and Allied Staff Association, Vancouver, Canada; ' assistant professor of surgery, Vancouver General Hospital, Vancouver, Canada and vice president of medical staff, Vancouver Medical, Dental and Allied Staff Association, Vancouver, Canada; ${ }^{\text {D }}$ consulting staff, Vancouver General Hospital, Vancouver, Canada and secretary medical staff, Vancouver Medical, Dental and Allied Staff Association, Vancouver, Canada; ${ }^{E}$ clinical assistant professor, University of British Columbia, medical director cardiac function clinic, Vancouver Hospital, Vancouver, Canada and chair advocacy committee, Vancouver Medical, Dental and Allied Staff Association, Vancouver, Canada; ${ }^{\text {F }}$ project and initiative manager, Facility Engagement Initiative, Vancouver, Canada; ${ }^{G}$ clinical instructor, University of British Columbia, medical microbiologist, Vancouver General Hospital, Vancouver, Canada; ${ }^{\mathrm{H}}$ clinical associate professor, University of British Columbia, staff neurologist, Vancouver General

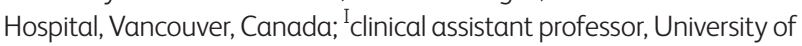
British Columbia, medical lead, Vancouver Coastal Health Laboratories, program director, UBC Medical Biochemistry Residency Training Program, Medical Biochemist, Vancouver General Hospital, Vancouver, Canada; ' Clinical professor, University of British Columbia, staff anaesthesiologist, Vancouver General Hospital, Vancouver, Canada
KEYWORDS: Physician engagement, physician burnout, patient care

\section{Introduction}

In the business world, employee engagement has become a central element of organisational management, not only because it reduces the costs of recruitment and retention but also it encourages and harnesses the development of new ideas that may produce a competitive advantage. These potential benefits also apply to the healthcare field where engagement of physicians with their healthcare community or institution can also improve patient care, enhance physician well-being and lead to safer, more effective and less costly healthcare. ${ }^{1-5}$ Engaged physicians are more productive than their less engaged colleagues. ${ }^{2,6}$ Organisations with physicians who find it rewarding and proud to be a part of their institution significantly outperform organisations with physicians missing these sentiments. ${ }^{2,7}$ Patient adherence to recommendations including adherence to medication, is associated with physician job satisfaction. ${ }^{3}$

Physicians who become engaged with a Hospital-Based Practice Improvement (Learning) Module are more likely to have successful experiences in implementing quality improvement in hospital settings than those who do not become engaged. ${ }^{8}$

In the Canadian context, a hospital that enhanced physician satisfaction found a dramatic improvement in clinical quality metrics. $^{9}$

There are a number of factors responsible for physician disengagement from the organisation. Some of the major factors are as follows. First, physicians' main role is in patient care and their expectation is that the organisation will provide sufficient resources for them to accomplish this goal. The absence of a physician voice in resource allocation may be interpreted by some organisational leaders as 'physician apathy'. In contrast, physicians may view situations of unsatisfactory provision of resources as 'organisational failure'. Second, physicians have a paramount responsibility to advocate for their patients and the patients' access to the best treatment. If a primary responsibility of their institution/hospital is cost containment, there is the potential for conflict. Third, the culture of the organisation may not support or welcome physician opinions. ${ }^{10}$ Fourth, physicians are currently under more stress than previously because of changes in the expectations from 
patients, and the healthcare system in which they operate. ${ }^{11}$ The requirements of being adherent to protocols, guidelines, audits and regulations creates a sense of loss of autonomy, ${ }^{11}$ especially in circumstances when the physician voice may have been minimal or absent from the development of protocols or policies. These factors lead to physician unhappiness which, in turn, adversely affects patient care. ${ }^{12}$

There are organisational characteristics that Kummerow and Kirby ${ }^{13}$ have identified that may contribute to physician disengagement, stress and burnout.

> Unemotionality - members do not express their real feelings. Problems are internalised, dealt at arm's length and left unresolved.

> Depersonalisation - human factors are depersonalised into management or committees.

$>$ Subordination - subordinates are made to suffer in silence and not challenge authority.

> Conservatism - individuals believe that things will never change and problem solving is half-hearted.

$>$ Isolationism - the organisation adopts individualistic rather than participative approach to decision making. Information is withheld and expertise is under-utilised.

> Antipathy - low overall cohesion and fragmentation into competing interests.

To accomplish the mission/goal of meaningful physician engagement, the Vancouver Medical, Dental and Allied Staff (VMDAS) Association executive and its committee on advocacy set about to establish a 'charter' for physician engagement. A social compact is a document that guides the relationship of its members between each other and other organisations. The concept of the 'charter' was stimulated by Vancouver Coastal Health community engagement initiative to engage patients in their health decision making. ${ }^{14}$ This charter has been circulated to the entire staff which includes over 1,900 members and was presented at the Medical Staff quarterly meeting. Feedback was taken into consideration during finalisation of the document. It has also been submitted to the senior management of the institution for their input. Recognising the attempts of other physicians to establish meaningful dialogue with their institutions' administration or senior management, ${ }^{15}$ we are presenting this document to potentially assist other organisations to develop an approach to improve patient care and physician well-being.

\section{The charter}

\section{What is meaningful physician engagement?}

The VMDAS Association has a mission/goal to foster meaningful physician representation to better serve our patients, our community of physicians and Vancouver Coastal Health. We aim to help create a culture at Vancouver Coastal Health where physicians have an active role in healthcare planning and decision making as well as in the provision of healthcare.

To be effective, physician engagement must be meaningful. This is characterised by:

> a physician's ability to influence decisions and affect the outcomes of programme development, delivery and management

$>$ broad and diverse physician community involvement

$>$ inclusivity and accessibility

> being respectful of physicians' schedules

> recognition of physicians' clinical schedule

$>$ advance notification of new projects and policies in a timeframe that permits optimal physician input

> balanced representation from all physician stakeholders who might be impacted by a decision or change

$>$ utilisation of methods of engagement and levels of participation tailored to the physician community and the project's needs.

\section{Why is meaningful engagement important?}

Facilities with higher levels of engagement have better patient outcomes and greater work satisfaction among physicians. ${ }^{7-9,16,17}$ Health systems face pressure to deliver quality healthcare and engage in continuous improvement while still containing costs. Physicians are one of the main professional groups who deliver care, and their direct experience equips them with knowledge and skills that can contribute to solving problems and coping with changing demands in their organisation.

\section{What does engagement look like in action?}

Vancouver Coastal Health engages physicians along a spectrum of participation that ranges from informing to empowering. Our goal is to support the greatest level of engagement possible and to build capacity, both within the organisation and within the physician community, to partner with the institution in order to achieve the best health outcomes for our patients (Table 1). ${ }^{18}$

\section{When should physician input be sought?}

When considering the question of 'when' to involve physicians, the timeline of the project is an important factor. These principles are similar to those outlined by other stakeholders. ${ }^{14}$ Physicians should be involved at the earliest stage possible when issues or opportunities are first identified. When physicians are added in later stages of a project, the project loses the valuable input of physicians to create the best possible outcomes. Involvement of physicians at the later stages of a project often means that they are less able to influence and improve the project, and results in feelings of being undervalued and disengagement.

Table 1. Engagement spectrum. Adapted with permission from International Association for Public Participation. IAP2 spectrum. Denver: IAP2

\section{Least engaging}

\section{Inform}

Physicians receive information and announcements

\section{Consult}

Physicians consulted on draft plans or on issues; feedback impacts decisions

\section{Involve}

Institution involves physicians with healthcare planning and policy processes

Most engaging

\section{Collaborate}

Physicians share decision making with the institution

\section{Empower}

Physicians identify issues, solutions and actions with institutional support 
Physician engagement can be invaluable in the following circumstances:

> development and/or drafting of policies, documents, programmes or projects

$>$ development of healthcare products or services

$>$ allocation of resources including facility design

> addressing the concerns of stakeholders and/or the general public ${ }^{14}$

building and improving two-way communication between physicians, healthcare institutions and the general community

> building capacity in the physician community to enable their effective participation in the healthcare system

> providing ongoing feedback on the implementation of a plan or initiative.

To be effective, physician engagement must be meaningful

Conditions of sub-optimal physician engagement include situations in which:

> physicians will not be able to influence decision makers (often referred to as 'tokenistic' engagement)

$>$ an institutional decision or direction has already been made or determined

$>$ an institutional decision or direction does not align with a decision/direction that physicians would support

$>$ there is inadequate support (time, compensation) available for physicians

$>$ there are inadequate physician voices on a committee or decision-making body

> physician representation is sought by group with a finite term which is entering its latter stages

> other members of a committee or decision-making group are not prepared to work with physicians.

Recognition of the time commitments and contribution of physicians

Physicians' work-life balance may limit the time available, as well as the length of time available, to contribute to engagement with the organisation.

> Recognising or thanking physicians for their suggestions or contribution to improving the organisations activities and mandate. The kind of recognition is not specified but would take into consideration the nature of the contribution.

> Physician members of the medical staff should be supportive of their physician colleagues in the medical staff.

The need for widespread physician engagement across the organisation, not just at the leadership level

While recognising the importance of physician leaders within the hierarchical system for efficacy of organisational management, it should also to be noted that physicians in formal executive positions are insufficient for developing physician engagement. More elaborate processes of engagement at the individual, organisational and system levels are necessary to support physician involvement in system improvement. ${ }^{19}$ The speed and extent of health reform demands shared leadership between physicians, the organisation's executives and among the breadth of individuals involved in healthcare. ${ }^{19}$
Clark et al concluded that

Physician engagement is not only about the appointment of a small group of leaders to roles such as medical or clinical director. It is recognition that leadership is a social function and not just defined by hierarchical reporting lines. Enhanced medical engagement should work towards a model of diffused leadership, where influence is exercised across relationships, systems and cultures. It should apply to all rather than a few. ${ }^{20}$

Physician leadership - at all levels - is required to improve physician engagement. ${ }^{21}$ It is essential the engagement should be a collaborative process between physicians and administration to form a strong alliance between 'clinicians and executives' emphasising a collaborative engagement between clinical and administrative staff. ${ }^{1}$ The net effect will be both an improvement in patient care and an improvement in physician 'job satisfaction'.

\section{Assessment of progress}

Establishment of a charter has been an essential step in the process of medical engagement, which has rightly been labelled 'a journey, not an event'. The charter has been used to engage the institution's senior leadership. Two conjoined events with institutional leaders and operations managers have occurred to inform and encourage engagement across the engagement spectrum. The subject matter is discussed at monthly meetings with members of senior leadership. Further dialogue is anticipated and is required to implement processes and structures to embed physician engagement at all levels.

There are structured instruments available to assess physician engagement. ${ }^{22}$ However, our institution has retained evaluators who are collecting data on the level of physician engagement that has developed with this process and to provide insight into its impact.

\section{Conclusion}

The development of this charter for physician engagement, embodies elements that have reinforced the importance to leadership of the benefits of physician engagement, not only for improved organisational management but also for more efficient and effective patient care. This initiative is of considerable value especially in times of funding constrains for healthcare expenditures. Physician engagement should lead to less 'physician burnout', improve physician recruitment and retention and have a positive effect on the relationship with other health professionals within the healthcare institution. There is a net benefit for all of us.

\section{Conflicts of Interest}

One co-author receives compensation as a paid assistant to the Vancouver Medical, Dental and Allied Staff Association

\section{Acknowledgments}

The authors would like to thank the members of the Vancouver Medical, Dental and Allied Staff Association and to thank the Vancouver Coastal Health's community engagement department that wrote the document How to engage patient and public advisors: a guide for staff. ${ }^{14}$

The authors would also like to acknowledge that 'Nothing about physicians, without physicians' is inspired by the slogan 'Nothing about us, without us,' used to convey the idea that no policy should be decided without participation from the members of the group(s) affected; this slogan, during the 1990s, rose to prominence among activists for people with disabilities. 


\section{References}

1 Milliken AD. Physician engagement: a necessary but reciprocal process. CMAJ 2014;186:244-5.

2 Owens K, Eggers J, Keller S, McDonald A. The imperative of culture: a quantitative analysis of the impact of culture on workforce engagement, patient experience, physician engagement, valuebased purchasing, and turnover. J Healthc Leadersh 2017:9:25-31.

3 DiMatteo MR, Sherbourne CD, Hays RD et al. Physicians' characteristics influence patients' adherence to medical treatment: results from the Medical Outcomes Study. Health Psychol 1993;12:93-102.

4 The King's Fund. Leadership and engagement for improvement in the NHS. London: The King's Fund, 2012. www.kingsfund.org.uk/sites/ default/files/field/field_publication_file/leadership-for-engagementimprovement-nhs-final-review2012.pdf [Accessed 08 March 2019].

5 Reinertsen J, Gosfield A, Rupp W, Whittington J. Engaging physicians in a shared quality agenda. Cambridge: Institute for Healthcare Improvement, 2007.

6 Shanafelt TD, Mungo M, Schmitgen ] et al. Longitudinal study evaluating the association between physician burnout and changes in professional work effort. Mayo Clin Proc 2016;91:422-31.

7 Clark J, Nath V. Medical engagement. A journey not an event. London: The King's Fund, 2014.

8 Caverzagie KJ, Bernabeo EC, Reddy SG, Holmboe ES. The role of physician engagement on the impact of the hospital-based practice improvement module (PIM). J Hosp Med 2009;4:466-70.

9 Studer Q, Hagins MJ, Cochrane BS. The power of engagement: creating the culture that gets your staff aligned and invested. Healthc Manag forum 2014;27:S79-97.

10 Taitz JM, Lee TH, Sequist TD. A framework for engaging physicians in quality and safety. BMJ Qual Saf 2012;21:722-8.

11 Edwards N, Kornacki MJ, Silversin J. Unhappy doctors: what are the causes and what can be done? BMJ 2002;324:835-8.

12 Grol R, Mokkink H, Smits A et al. Work satisfaction of general practitioners and the quality of patient care. Fam $\operatorname{Pr} 1985 ; 2: 128-35$.
13 Kummerow E, Kirby N. Organisational culture: concept, context, and measurement. World Scientific Pub Co Inc, 2013.

14 Vancouver Costal Health. How to engage patient and public advisors: a guide for staff. Vancouver Costal Health. http://mpap.vch. ca/wp-content/uploads/sites/26/2017/07/How-to-Engage-Patientand-Public-Advisors-A-Guide-for-Staff-FINAL2.pdf.

15 Scott CG, Theriault A, McGuire S et al. Developing a physician engagement agreement at The Ottawa Hospital: a collaborative approach. Healthc Q 2012;15:50-3.

16 Nelson MF, Merriman CS, Magnusson PT et al. Creating a physician-led quality imperative. Am J Med Qual 2014;29:508-16.

17 Marsden J, van Dijk M, Doris P, Krause C, Cochrane D. Improving care for British Columbians: the critical role of physician engagement. Healthc Q 2012;15:51-5.

18 International Association for Public Participation. IAP2 Spectrum. Denver: IAP2. https://iap2canada.ca/Resources/Documents/0702Foundations-Spectrum-MW-rev2\% 20(1).pdf.

19 Dickson G, Tholl B, Baker GR et al. Partnerships for health system improvement (PHSI) leadership and health system redesign: crosscase analysis final report. Royal Road University and Canadian Health Leadership Network (CHLNet), 2014.

20 Clark J, Spurgeon P, Hamilton P. Medical professionalism: Leadership competency_An essential ingredient. Int J Clin Leadersh 2008;16:3-9.

21 Van Aerde J, Dickson G. Accepting our responsibility. A blueprint for physician leadership in transforming Canada's health care system. Ottawa: CSPL/SCLM, 2017.

22 NHS Grampian (Acute). Medical engagement scale. NHS, 2014.

Address for correspondence: Dr Simon Rabkin, Department of Medicine (Cardiology) Vancouver General Hospital, University of British Columbia, 2775 Laurel Street, Vancouver, British Columbia V6T 1Z4, Canada.

Email:simon.rabkin@ubc.ca

\section{4": Royal College of Physicians}

\section{Advancing medical professionalism}

Articulating a modern professional identity helps doctors to understand and undertake the unique role they play in healthcare. Developed in consultation with healthcare professionals, patients and other stakeholders, this report from the Royal College of Physicians aims to help doctors improve their professionalism in practical ways.

It focuses on seven key aspects of professional practice:

$>$ doctor as healer

$>$ patient partner

$>$ team worker

$>$ manager and leader

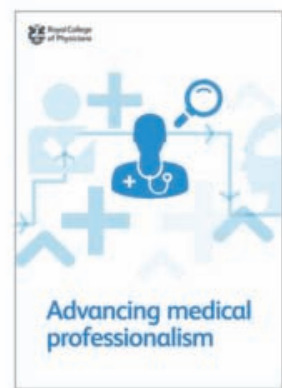

Download the report at: www.rcplondon.ac.uk/advancing-medical-professionalism 